\title{
THE Onfp CLASS IN THE MAGELLANIC CLOUDS
}

\author{
Nolan R. Walborn ${ }^{1,12}$, Ian D. Howarth ${ }^{2,13}$, Christopher J. Evans ${ }^{3,13,14}$, Paul A. Crowther ${ }^{4,14}$, Anthony F. \\ J. Moffat ${ }^{5,12}$, Nicole St-Louis ${ }^{5}$, Cecilia Fariña ${ }^{6,7}$, Gulllermo L. Bosch ${ }^{6,7,15}$, Nidia I. Morrell ${ }^{8}$, Rodolfo H. Barba A $^{-9.11,15}$, \\ AND JACCO TH. VAN LOON ${ }^{11}$ \\ ${ }^{1}$ Space Telescope Science Institute ${ }^{16}, 3700$ San Martin Drive, Baltimore, MD 21218, USA; walborn@stsci.edu \\ ${ }^{2}$ Department of Physics and Astronomy, University College London. Gower Street. London WC1E 6BT. UK; idh@star.ucl.ac.uk \\ ${ }^{3}$ UK Astronomy Technology Centre, Royal Observatory Edinburgh. Blackford Hill. Edinburgh EH9 3HJ, UK; chris.evans@stfc.ac.uk \\ ${ }^{4}$ Department of Physics and Astronomy, University of Sheffield, Hounsfield Road, Sheffield S3 7RH, UK; Paul.Crowther@sheffield.ac.uk \\ ${ }^{5}$ Département de Physique, Université de Montreal, C.P. 6128, Succ. Centre-Ville, Montreal, QC H3C 3J7. Canada; moffat@astro.umontreal.ca, \\ stlouis@astro.umontreal.ca \\ ${ }^{6}$ Facultad de Ciencias Astronómicas y Geofísicas. Universidad Nacional de La Plata. 1900 La Plata. Argentina; ceciliaf@fcaglp.unlp.edu.ar. \\ guille@fcaglp.unlp.edu.ar \\ ${ }^{7}$ IALP-CONICET, Argentina \\ ${ }^{8}$ Las Campanas Observatory, Observatories of the Carnegie Institution of Washington. Casilla 601, La Serena, Chile; nmorrell@ @ico.cl \\ ${ }^{9}$ Departamento de Física. Universidad de La Serena. Cisternas 1200 Norte, La Serena. Chile; rbarba@ dfuls.cl \\ ${ }^{10}$ Instituto de Ciencias Astronómicas de la Tierra y del Espacio (ICATE-CONICET). Avenida España 1512 Sur. J5402DSP San Juan. Argentina \\ ${ }^{11}$ Astrophysics Group, School of Physical \& Geographical Sciences, Keele University, Staffordshire ST5 5BG, UK; jacco@astro.keele.ac.uk \\ Received 2009 October 1; accepted 2010 January 14; published 2010 February 11
}

\begin{abstract}
The Onfp class of rotationally broadened, hot spectra was defined some time ago in the Galaxy, where its membership to date numbers only eight. The principal defining characteristic is a broad, centrally reversed He II $\lambda 4686$ emission profile; other emission and absorption lines are also rotationally broadened. Recent surveys in the Magellanic Clouds (MCs) have brought the class membership there, including some related spectra, to 28 . We present a survey of the spectral morphology and rotational velocities, as a first step toward elucidating the nature of this class. Evolved, rapidly rotating hot stars are not expected theoretically, because the stellar winds should brake the rotation. Luminosity classification of these spectra is not possible, because the principal criterion (He in $\lambda 4686$ ) is peculiar; however, the MCs provide reliable absolute magnitudes, which show that they span the entire range from dwarfs to supergiants. The Onfp line-broadening distribution is distinct and shifted toward larger values from those of normal O dwarfs and supergiants with $>99.99 \%$ confidence. All cases with multiple observations show line-profile variations, which even remove some objects from the class temporarily. Some of them are spectroscopic binaries; it is possible that the peculiar profiles may have multiple causes among different objects. The origin and future of these stars are intriguing; for instance, they could be stellar mergers and/or gamma-ray-burst progenitors.
\end{abstract}

Key words: Magellanic Clouds - stars: early-type - stars: emission-line, Be - stars: fundamental parameters stars: rotation - stars: variables: general

Online-only material: color figures

\section{INTRODUCTION}

The Onfp designation was introduced by Walborn (1973) to describe several peculiar ("p") early-type spectra; the " $n$ " is the classical descriptor of broadened absorption lines, generally ascribed to rapid rotation, while " $\mathrm{f}$ " denotes O-type spectra with He II $\lambda 4686$ and $\mathrm{N}$ III $\lambda \lambda 4634-464()-4642$ in selective (Walborn 2001) emission. The defining peculiarity consists of a composite emission + absorption profile at $\lambda 4686$, which ranges among different objects from a strong absorption line with weak emission wings to a strong, broad emission line with a central absorption reversal. The class was independently and simultaneously recognized by Conti \& Leep (1974), who designated it Oef, by implied analogy with the Be stars.

\footnotetext{
${ }^{12}$ Visiting Astronomer, Cerro Tololo Inter-American Observatory, National Optical Astronomy Observatory, operated by the Association of Universities for Research in Astronomy. Inc., under a cooperative agreement with the NSF.

13 Visiting Astronomer, Anglo-Australian Observatory.

14 Visiting Astronomer. European Southern Observatory.

15 Visiting Astronomer, Las Campanas Observatory, Observatories of the Carnegie Institution of Washington.

16 Operated by the Association of Universities for Research in Astronomy, Inc., under NASA contract NAS5-26555.
}

It is noteworthy that the two brightest Of stars in the sky, $\lambda$ Cephei (Walborn 1973; Conti \& Frost 1974) and $\zeta$ Puppis (Conti $\&$ Niemela 1976), belong to this peculiar class. Contrary to what might be expected from that datum, modern classifications of about 400 Galactic $O$ stars have yielded only six additional members (Maíz Apellániz et al. 2004). Thus, they are rare objects. The known Galactic Onfp spectra are listed in Table 1 with references.

Extensive recent studies and surveys of the rich O-type population of the Magellanic Clouds (MCs) have yielded an increasing sample of Onfp and related spectra, which provide the motivation and subject of this work. A larger sample with known distances may provide vital information toward a physical interpretation of the class. The number of objects available for discussion here is 28 . However, it should not be inferred that they have a higher incidence in the MCs; e.g., Evans et al. (2004) found just one among $139 \mathrm{O}$ stars in their study, and the current survey by I. D. Howarth comprises 191 O stars among which there are four new Onfp spectra. These objects are included in the present sample.

Mihalas \& Conti (1980) proposed that the phenomenology of $\zeta$ Pup might result from co-rotation of the inner stellar wind enforced by a weak (50 gauss) magnetic field. From spec- 
Table 1

Galactic Onfp Spectra

\begin{tabular}{|c|c|c|c|c|c|}
\hline Name & Sp Type & $V$ & $B-V$ & $\begin{array}{c}v \sin i \\
\left(\mathrm{~km} \mathrm{~s}^{-1}\right)\end{array}$ & References \\
\hline$\zeta$ Pup & O4 I(n)fp & 2.25 & -0.28 & 219 & Conti \& Niemela 1976 \\
\hline$\lambda$ Cep & $06 \mathrm{I}(\mathrm{n}) \mathrm{fp}$ & 5.05 & 0.24 & 219 & Conti \& Frost 1974 \\
\hline HD 14434 & $05.5 \mathrm{Vn}((\mathrm{f})) \mathrm{p}$ & 8.49 & 0.17 & 423: & Kendall et al. 1995, 1996; De Becker \& Rauw 2004 \\
\hline HD 14442 & $05 n(f) p$ & 9.22 & 0.41 & 273 & Kendall et al. 1995, 1996; De Becker \& Rauw 2004 \\
\hline HD 152248 & O7 Ib:(n)(f)p & 6.13 & 0.14 & $159+165$ & Sana et al. 2001; Mayer et al. 2008 \\
\hline HD 172175 & O6 I (n)fp & 9.44 & 0.64 & $\ldots$ & Walborn 1982 ; A. Sota et al. 2010 (in preparation) \\
\hline HD 192281 & O5 Vn((f))p & 7.55 & 0.38 & 270 & De Becker \& Rauw 2004 \\
\hline $\mathrm{BD}+60^{:} 2522$ & $06.5(\mathrm{n})(\mathrm{f}) \mathrm{p}$ & 8.66 & 0.40 & $178-240$ & Rauw et al. 2003 \\
\hline
\end{tabular}

Table 2

Onfp and Related Spectra in the MCs

\begin{tabular}{|c|c|c|c|c|c|c|c|c|c|}
\hline Name $^{\mathrm{a}}$ & R.A. (2000) & Decl. (2000) & Sp Type & $V$ & $B-V$ & $\operatorname{Ref}^{b}$ & $M_{V}$ & $\begin{array}{c}v \sin i \\
\left(\mathrm{~km} \mathrm{~s}^{-1}\right)\end{array}$ & Spectroscopic Reference \\
\hline AV 80 & 05043.8 & -724741 & $04-6 n(f) p$ & 13.33 & -0.14 & 1 & -6.3 & 370 & Walborn et al. 2000 \\
\hline AV 321 & 10257.1 & -720809 & O9 IInp & 13.88 & -0.21 & 1 & -5.5 & 280 & P. A. Crowther (prev. unpubl.) \\
\hline Sk 190 & 13128.0 & -732214 & $07.5 n(f) p$ & 13.59 & -0.22 & 2 & -5.8 & 320 & P. A. Crowther (prev. unpubl.) \\
\hline 2dFS 3975 & 13128.0 & -732214 & $O 8 n(f) p$ & 13.54 & -0.18 & 3 & -6.0 & 340 & Evans et al. 2004 \\
\hline $\mathrm{N} 11-20$ & 45650.3 & -663104 & $\mathrm{O} 5 \mathrm{Inf}+\mathrm{p}$ & 13.18 & -0.22 & 4 & -5.7 & 260 & Evans et al. 2006 \\
\hline Sk $-69^{\circ} 50$ & 45715.1 & -692020 & $O 7(n)(f) p$ & 13.26: & -0.13 & 5 & $-5.9:$ & 210 & P. A. Crowther (prev. unpubl.) \\
\hline $\mathrm{AA} \Omega \mathrm{N} 11-87$ & 45844.9 & -661215 & $06.5(\mathrm{n}) \mathrm{fp}$ & 13.12 & -0.30 & 6 & -5.5 & 180 & C. J. Evans (prev. unpubl.) \\
\hline $2 \mathrm{dFL} 51-106$ & 51849.5 & -691406 & O7(n)fp & 12.84 & -0.14 & 7 & -6.3 & 140 & I. D. Howarth (prev. unpubl.) \\
\hline Sk $-65=47$ & 52054.7 & -652718 & $\mathrm{O} 4 \mathrm{I}(\mathrm{n}) \mathrm{f}+\mathrm{p}$ & 12.51 & -0.18 & 8 & -6.5 & 200 & P. A. Crowther (prev. unpubl.) \\
\hline Sk $-67^{-} 111$ & 52648.1 & -672930 & O6 Ia(n)fpv & 12.57 & -0.20 & 8 & -6.4 & 230 & Walborn et al. $2002 \mathrm{a}$ \\
\hline $2 \mathrm{dFL} 52-50$ & 52901.7 & -683204 & O7 Ianf & 13.26 & -0.15 & 6 & -5.9 & 230 & I. D. Howarth (prev. unpub1.) \\
\hline $2 \mathrm{dFL}, 51-50$ & 53108.1 & -683654 & $\mathrm{O} 7.5(\mathrm{n})(\mathrm{f}) \mathrm{p}$ & 12.82 & -0.18 & 6 & -6.2 & 270 & I. D. Howarth (prev. unpub1.) \\
\hline HDE 269702 & 53152.1 & -673421 & $O 8 I(f) p$ & 12.08 & -0.17 & 8 & -6.9 & 110 & P. A. Crowther (prev. unpubl.) \\
\hline $\mathrm{AA} \Omega 2$ 30Dor 142 & 53406.3 & -692509 & $06.5(\mathrm{n})(\mathrm{f}) \mathrm{p}$ & 13.24 & -0.15 & 6 & -5.9 & 210 & C. J. Evans (prev. unpubl.) \\
\hline $\mathrm{AA} \Omega 230 \mathrm{DOI} 187$ & 53551.9 & -692319 & O6n $(f) p$ & 13.40 & -0.18 & 6 & -5.6 & 350 & C. J. Evans (prev. unpubl.) \\
\hline $\mathrm{ST} 2-32$ & 53555.4 & -691200 & O5n(f)p & 13.95 & 0.09 & 9 & -5.9 & $\cdots$ & A. F. J. Moffat (prev. unpubl.) \\
\hline ST $2-53$ & 53606.4 & -691148 & O5n(f)p & 12.29 & -0.15 & 9 & -6.8 & $\cdots$ & A. F. J. Moffat (prev. unpubl.) \\
\hline ST $1-28$ & 53738.0 & -691015 & O3.5 Infp & 14.26 & 0.18 & 9 & -5.8 & $\ldots$ & A. F. J. Moffat (prev. unpubl.) \\
\hline Brey $73-2 A$ & 53746.6 & -690908 & $\mathrm{O} 4(\mathrm{n})(\mathrm{f}) \mathrm{p}$ & 14.13 & 0.13 & 10 & -5.8 & $\cdots$ & Walborn et al. 1999 \\
\hline ST $1-93$ & 53756.2 & -691151 & O6 Ianfp & 14.88 & 0.27 & 9 & -5.5 & $\cdots$ & A. F. J. Moffat (prev. unpubl.) \\
\hline Parker 841 & 53841.2 & -690552 & $\mathrm{O} 4-6(\mathrm{n})(\mathrm{f}) \mathrm{p}$ & 15.18 & 0.17 & 11 & -4.9 & .. & Walborn \& Blades 1997 \\
\hline $\mathrm{AA} \Omega \Omega 30 \mathrm{Dor} 320$ & 53846.0 & -692837 & $O 7 n(f) p$ & 13.75 & 0.05 & 6 & -6.0 & 300 & C. J. Evans (prev. unpubl.) \\
\hline$A A \Omega 30 D$ or 333 & 53911.6 & -693037 & $\mathrm{O} 2-3(\mathrm{n}) \mathrm{f} * \mathrm{p}$ & 12.27 & 0.10 & 6 & -7.6 & 170 & C. J. Evans (prev. unpubl.) \\
\hline Fariña 35 & 53938.9 & -694436 & O8(f)p & 14.61 & -0.08 & 6 & -4.7 & $<100$ & Fariña et al. 2009 \\
\hline Fariña 72 & 53959.8 & -693611 & $06 n(f+) p$ & 13.75 & -0.04 & 6 & -5.7 & 150 & Fariña et al. 2009 \\
\hline Fariña 82 & 54004.6 & -693951 & O5n $(f+) p$ & 12.38 & -0.03 & 6 & -7.1 & 280 & Fariña et al. 2009 \\
\hline $\mathrm{AA} \Omega 2$ 30Dor 368 & 54013.8 & -692535 & $07.5 n(f) p$ & 13.30 & -0.12 & 6 & -5.9 & 280 & C. J. Evans (prev. unpubl.) \\
\hline Fariña 151 & 54109.8 & -693916 & $O 7 n(f) p$ & 13.21 & 0.19 & 6 & -6.9 & 270 & Fariña et al. 2009 \\
\hline
\end{tabular}

Notes.

a AV: Azzopardi \& Vigneau 1982; Sk: Sanduleak 1969, 1970; N: Henize 1956; AAS2: J. van Loon et al. unpublished survey; 2dFL: I. D. Howarth unpublished survey; ST: Schild \& Testor 1992; Brey: Breysacher et al. 1999; Parker: Parker 1993.

b Photometric References: (1) Azzopardi \& Vigneau 1982; (2) Ardeberg 1980; (3) Massey 2002; (4) Evans et al. 2006; (5) Isserstedt 1979; (6) Zaritsky et al. 2004; (7) Udalski et al. 2000; (8) Isserstedt 1975; (9) Schild \& Testor 1992; (10) Walborn et al. 1999; (11) Parker 1993.

trophotometry of the variable $\mathrm{H} \alpha$ profile, Moffat \& Michaud (1981) suggested that $\zeta$ Pup might be a magnetic oblique rotator with a period of about 5 days. However, J.-F. Donati \& I. D. Howarth (unpublished) were unable to detect a field on this star to a comparable limit, albeit orientation dependent. Henrichs (1991) reported correlated $\lambda 4686$ and UV wind-profile variations, possibly periodic, in $\lambda$ Cephei on very short timescales, and he inferred that non-radial pulsations may be present. The similarity of the Onfp He II $\lambda 4686$ profiles to the Balmer profiles of Be stars might suggest formation in a disk. However, Bouret et al. (2008) have successfully modeled the $\lambda 4686$ and
$\mathrm{H} \alpha$ profiles of $\zeta$ Pup with a clumped, rotating wind alone. We shall present available and suggest future observations that may eventually elucidate the origin(s) of the peculiar Onfp spectral morphology.

\section{OBSERVATIONS}

Information about the stars discussed here is presented in Table 2; the first three are in the Small Magellanic Cloud (SMC), and the remaining 25 are in the Large Magellanic Cloud (LMC). (There are two independent observations for two stars: Sk $190=$ 
$2 \mathrm{dFS} 3975$ in the SMC, and 2dFL 52-171= AA $\Omega$ 30Dor 142 in the LMC.) In this section, we provide observational parameters of the diverse data sources, with more detail for cases in which that information is not available in references listed in the table.

Previously unpublished data of P. A. Crowther are from the UV-Visual Echelle Spectrograph (UVES) at the European Southern Observatory (ESO) Very Large Telescope (VLT). They were obtained under program 074.D-0109 during 2004 November 23-30. The slit width was 1:.2 and the UVES blue arm with a central setting of $4370 \mathrm{~A}$ provided continuous coverage of $3730-5000 \mathrm{~A}$ at a resolving power of 37,500 on a single $2 \times 4 \mathrm{~K}$ EEV CCD. The observation of AV 80 in the SMC used here was also obtained during that run, although an earlier observation is referenced in Table 2 . The observation of $S k-67^{\circ} 111$ is from a prior UVES run as specified by Walborn et al. (2002a).

The VLT FLAMES/Giraffe data of Evans et al. (2006) have a resolving power of 23,300 at He $п \lambda 4686$.

The 2-degree Field LMC (2dFL) survey by Howarth was conducted with the $2 \mathrm{dF}$ fiber-fed multi-object spectrographs on the $3.9 \mathrm{~m}$ Anglo-Australian Telescope; observations discussed here were obtained during the nights of 2004 December 4 and 8. Each of the two spectrographs was used with a 1200B grating, giving a resolution of $2 \AA$ (dispersion $1.1 \mathrm{~A} \mathrm{pixel}^{-1}$ ) over a wavelength range of $380(0-490)(\mathrm{A}$. The same system was used for the 2dFS survey in the SMC (Evans et al. 2004).

AAOmega (AA $\Omega$ ) was an upgrade of the $2 \mathrm{dF}$ spectrographs to dual-beam systems with new fiber feeds, using the same front-end optics. A pilot study in the LMC, led by van Loon (with Evans observing, hence "C. J. Evans prev. unpubl." in the table), was undertaken during 2006 February 22-24; only data from the blue arm are discussed here. On the first night fields were observed in 30 Dor and N11 with a $1700 \mathrm{~B}$ grating and two central wavelengths ( 4100 and $4700 \mathrm{~A}$ ), yielding complete blue coverage of 3765-4985 A, at a resolution of $1 \mathrm{~A}$ (dispersion $0.33 \mathrm{~A} \mathrm{pixel}^{-1}$ ). On the second night, the 30 Dor field was also observed with a $1500 \mathrm{~V}$ grating at a central wavelength of $4375 \AA$, delivering coverage of $3975-4755 \mathrm{~A}$ at a resolution of $1.25 \mathrm{~A}$ (dispersion $\sim 0.4 \mathrm{~A} \mathrm{pixel}^{-1}$ ).

Unpublished observations by A. F. J. Moffat are from the Argus multifiber spectrograph at the Cerro Tololo Inter-American Observatory (CTIO) $4 \mathrm{~m}$ telescope, as further specified by Walborn et al. (2002b); the spectral resolution is $2 \mathrm{~A}$.

The Hubble Space Telescope/Faint Object Spectrograph data of Walborn et al. (1999) have a dispersion of 3 A per diode, with a factor of 4 oversampling. They were smoothed by 3 pixels to a formal resolution of $2.25 \mathrm{~A}$ in that study; the representation of Brey 73-2A shown there has better fixed-pattern noise suppression than the one available here. The Anglo Australian Observatory/Royal Greenwich Observatory spectrograph (with fiber feed) data of Walborn \& Blades (1997) have a resolution of $2.2 \mathrm{~A}$ with relatively low signal-to-noise ratio $(\mathrm{S} / \mathrm{N})$ and heavy nebular-line contamination

The observations by Fariña et al. (2009) are from the Las Campanas Observatory $2.5 \mathrm{~m}$ telescope with the Wide Field Re-imaging CCD Camera in a multislit/grism configuration; the spectral resolution is $3.1 \mathrm{~A}$.

\section{RESULTS}

\subsection{Spectral Morphology}

Spectrograms of all these stars are displayed in Figures 1-3, in order of advancing spectral type. Note that all have been uniformly smoothed and rebinned as specified in the caption to
Figure 1. All of the classifications have been either newly derived or re-examined for consistency in this work. Two salient characteristics of the Onfp class are immediately apparent. First, as in the smaller Galactic sample, there is a substantial range in the appearance of the peculiar He II $\lambda 4686$ profiles, from strong emission with a small absorption reversal, to a dominant absorption feature with weak emission wings; and intermediate cases occur as well. Second, there is also a wide range of line broadening, with a predominance of large values, which affects both the absorption and emission lines equally again as in the Galactic counterparts. These properties suggest rapid rotation, in which the region producing the emission lines participates, with the minority smaller values plausibly arising from high inclinations.

The absorption to emission progression in the He II $\lambda 4686$ line of normal O-type spectra has been identified as a luminosity criterion (Walborn 1971, 1973, 2009; Walborn \& Fitzpatrick 1990). Clearly it would be hazardous to apply that inference to the peculiar Onfp profiles. Conservatively, only spectra with a strong, dominant emission feature have been given luminosity class I here, while no luminosity class has been assigned otherwise; cf. $\lambda$ Cep versus BD $+60^{\circ} 2522$ in Walborn (1973). The physical luminosities of these stars are investigated below by means of the $\mathrm{MC}$ distance moduli.

The comparable broadening of the absorption and emission lines, including $\mathrm{N}$ III $\lambda \lambda 4634-464()-4642$. is in keeping with the selective nature of the latter (Walborn 2001) and the evidence that they are predominantly of photospheric origin.

Comments on several of the individual spectra and related information follow. In several cases, they are relevant to subsequent discussion of possible origins of the peculiar profiles and their temporal variations.

$A A \Omega 30 D$ or 333 . It is possible that this very early spectrum does not belong in the Onfp category, but rather $\lambda 4686$ has a "composite" P Cygni profile (with weaker emission blueward of the absorption component) that is characteristic of these extreme spectral types (Walborn et al. 2002b). However, it is included here for completeness, since it satisfies the formal Onfp definition, and there could be a physical relationship. This star is 5-31 of Testor \& Niemela (1998) in the H II region N158 south of 30 Doradus, also discussed by Massey et al. (2000) and Walborn et al. (2002b). Its image is clearly extended, indicating a multiple system to which the extremely bright absolute magnitude in Table 2 refers.

Brey 73-2A. This star is a possible blue straggler in a compact cluster (Walborn et al. 1999) within the 30 Doradus B association (Schild \& Testor 1992), which might represent a clue to the origin of the Onfp class, as further discussed later. (ST 1-28 and ST 1-93 are in the same association, the former also in a subclustering.)

$A V$ 80. The apparent broad N IV $\lambda 4(058$ line in the optical observation shown by Walborn et al. (2000), which led to the spectral-type range given there, is much weaker if present at all in the UVES data shown here. Further observations are required to determine whether a real spectral variation is involved. Walborn et al. (2000) also showed the ultraviolet spectrum of this star; it has strong wind features as expected for its spectral type. Rapid rotation is not expected in evolved, massive stars with strong winds, which should be braked, as further discussed below. A quantitative analysis of this spectrum was performed by Heap et al. (2006).

Parker 841. This star is in the 30 Doradus core cluster (Walborn \& Blades 1997), which may indicate a very young object, unlike the majority of the Onfp class. 


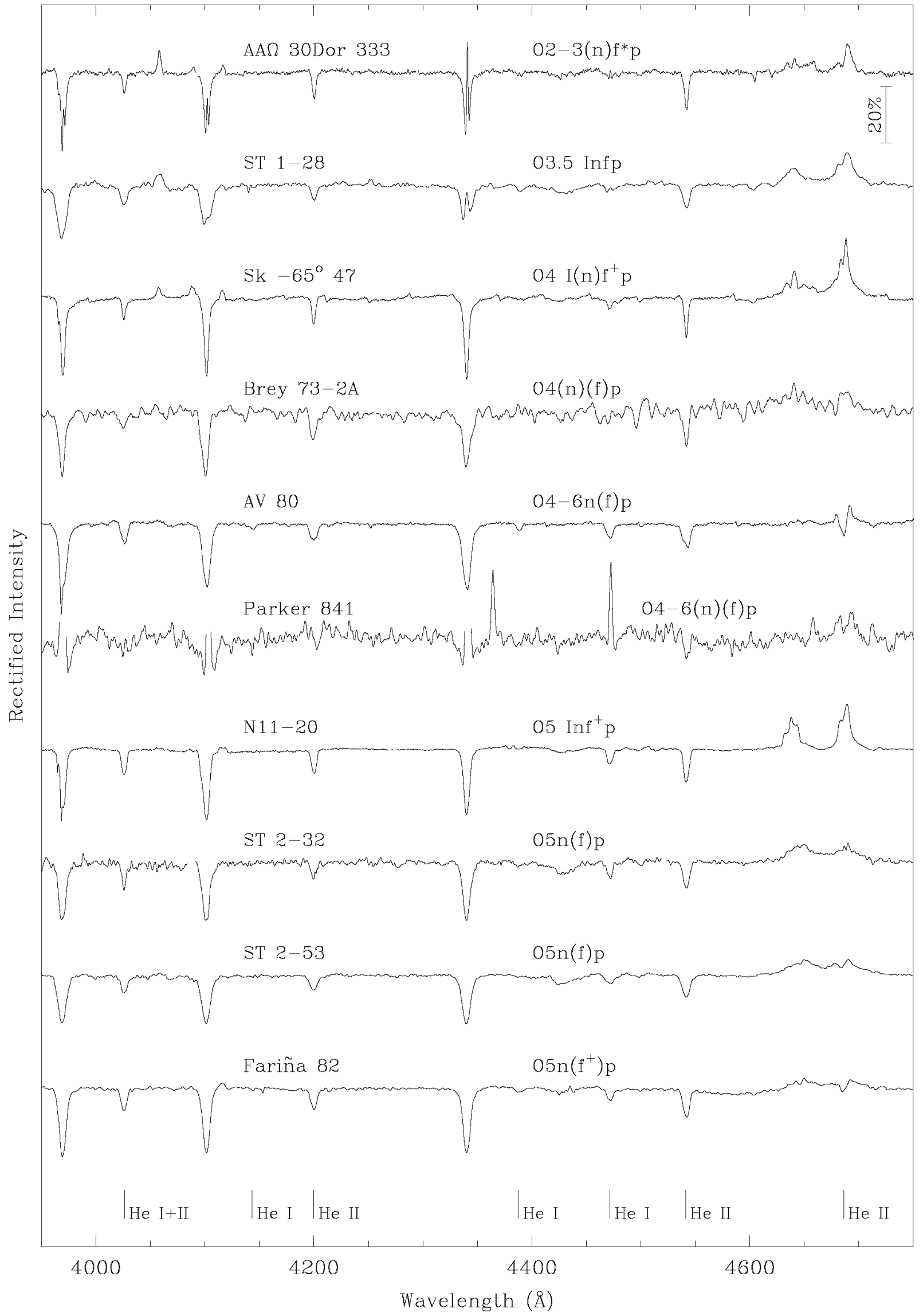

Figure 1. Rectified blue-green digital observations of MC Onfp spectra, in order of advancing spectral type. The original data have a variety of resolutions; all have been smoothed with an $0.3 \AA$ Gaussian and rebinned here. Adjacent spectrograms are offset by 0.4 continuum unit; the vertical bar at upper right denotes 0.2 continuum unit. Rest wavelengths are plotted. The identified lines are He I+II $\lambda 4026$; He I $\lambda \lambda 4144,4387,4471$; He II $\lambda \lambda 4200,4541,4686$. 


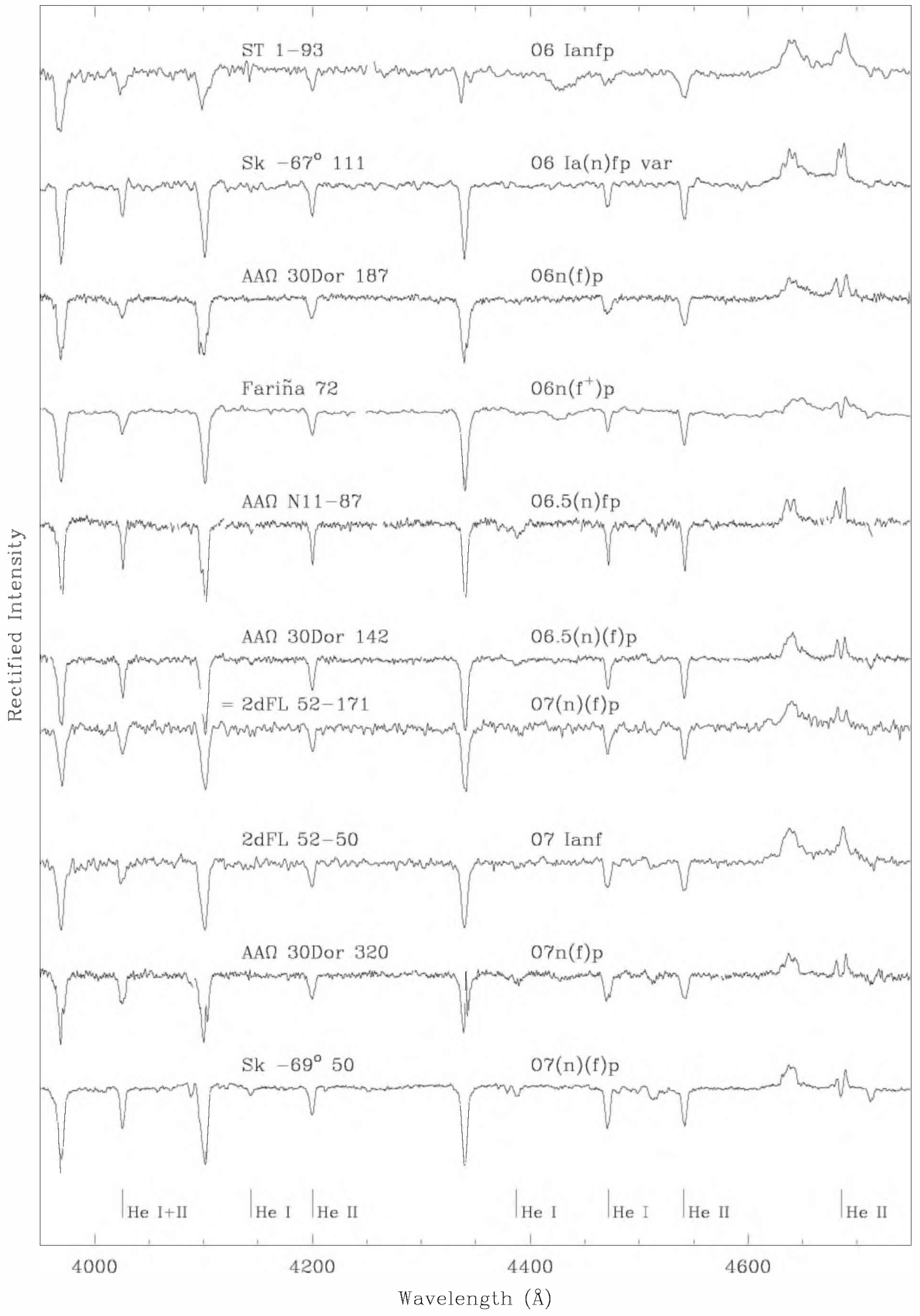

Figure 2. MC Onfp spectral-type sequence continued. See the caption to Figure 1. 


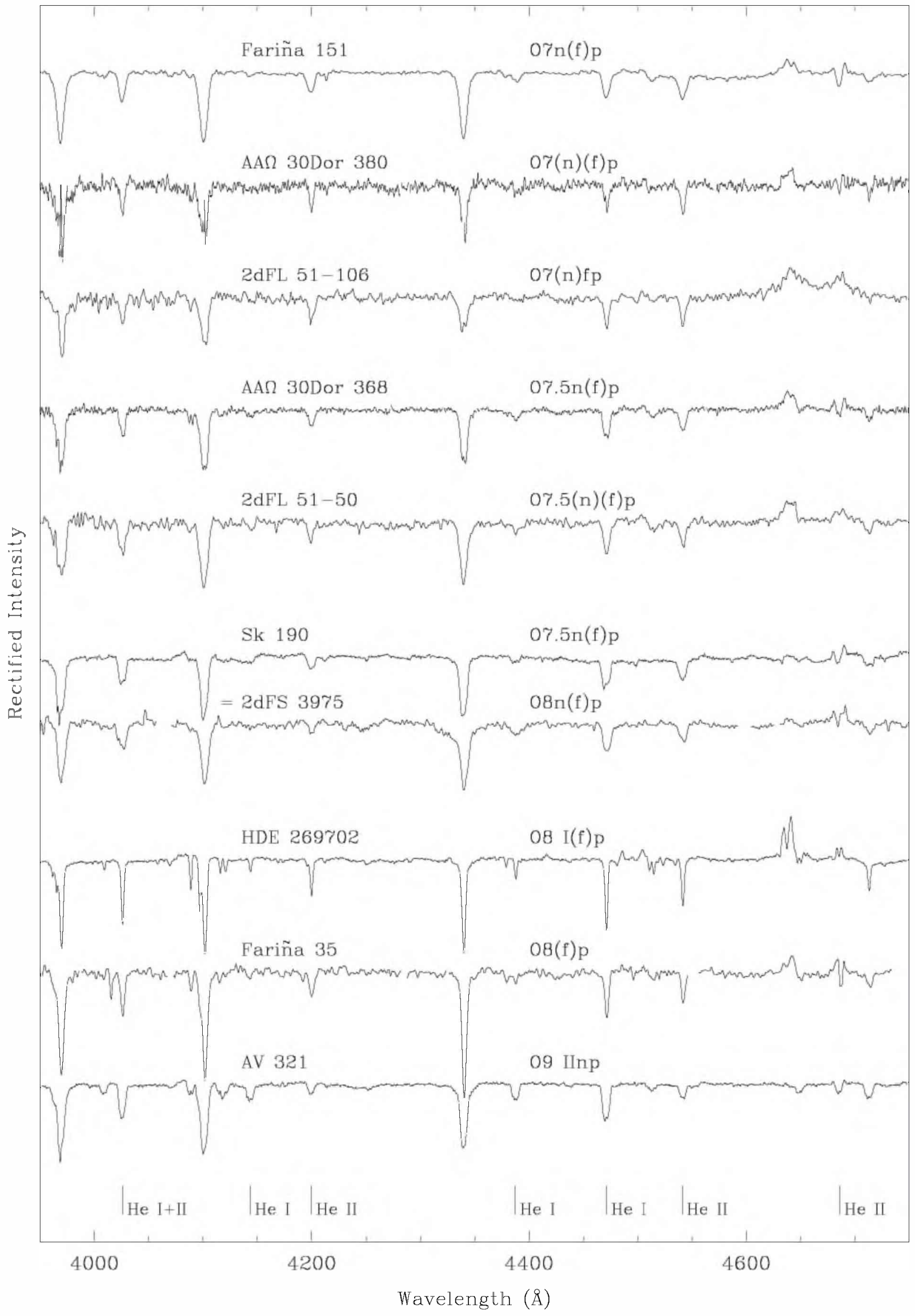

Figure 3. MC Onfp spectral-type sequence concluded. See the caption to Figure 1. 
ST 2-32 and ST 2-53. These two very similar spectra correspond to objects within 30 Doradus $\mathrm{C}$ (Schild \& Testor 1992); the latter is one of the brightest stars in the region and is in a subclustering.

$2 d F L 52-50$. The $\lambda 4686$ emission is single peaked, so this is not an Onfp spectrum. However, both absorption and emission lines of this apparent supergiant are unusually broadened, and as will be seen below, some Onfp objects with multiple observations intermittently remove themselves from the class, so this may well be a related object.

$A A \Omega 30$ Dor 320 . This star is 5-82 of Testor \& Niemela (1998).

$H D E$ 269702. This highly unusual supergiant spectrum has narrow lines and is one of only two in the sample without the " $n$ " qualifier in its type; it also has the lowest value of $v \sin i$ measured here (as described below). The very narrow, double $\lambda 4686$ emission is unique to date, but again, the correlation between absorption- and emission-line widths is typical of the Onfp class. Note that this observation is from UVES, and that this profile would likely not be detected in low-resolution data.

Fariña 35. This is the X-ray binary LMC X-1 (references in Fariña et al. 2009), so it is likely that the composite $\lambda 4686$ profile arises at least partly from systemic phenomena and not solely from the $\mathrm{O}$-star extended atmosphere. Nevertheless, it is retained in the present sample because it was selected from the survey data without knowledge of its identity. It is the other relatively narrow-lined spectrum in this sample. As shown by van der Meer et al. (2007-their online Figure 2), doublepeaked $\lambda 4686$ profiles are common at certain phases in $\mathrm{X}$ ray binaries, including SMC X-1 and LMC X-4. Evans et al. (2004) reported both double- and single-peaked $\lambda 4686$ profiles in different observations of SMC X-1; see also Lennon (1997).

$A V$ 321. Another unusual high-luminosity, very broad-lined spectrum. There is no "f" parameter in the spectral type because such is not used later than O8.5. The weak emission wings at $\lambda 4686$ may appear difficult to discern at the scale displayed here, but they are striking in the original high-resolution, highS/N UVES data. Again, this profile would likely not be detected in low-resolution data.

\subsection{Absolute Magnitudes}

Absolute visual magnitudes of the sample stars have been calculated from the photometry, with the assumptions of distance moduli of 18.6 for the LMC and 19.1 for the SMC, a value of $R=3.0$ for the ratio of total to selective extinction, and intrinsic colors of the spectral types as listed by Walborn et al. (2002a). The results are given in Table 2 and plotted in Figure 4 together with the luminosity-class calibration of Walborn (1973), updated for the O2-O3 stars by Walborn et al. (2002b). As shown by the latter and references cited therein, the $O$ spectral types are insensitive to metallicity since they depend upon the He ionization ratio, and there is no evidence for systematic differences among the visual absolute-magnitude calibrations of the Galactic and MC O spectral types, within the random uncertainties further discussed below.

It is immediately apparent that there is no absolute-magnitude distinction between the objects assigned luminosity class I and the others in this Onfp sample. Indeed, the isolation of peculiar objects is a pre-requisite for the recognition of trends and correlations among normal spectra.

Of course, objects that appear "too bright" may be unresolved multiple systems, particularly at the distance of the MCs (e.g., Walborn et al. 1999). Inversely, for an assumed distance, the

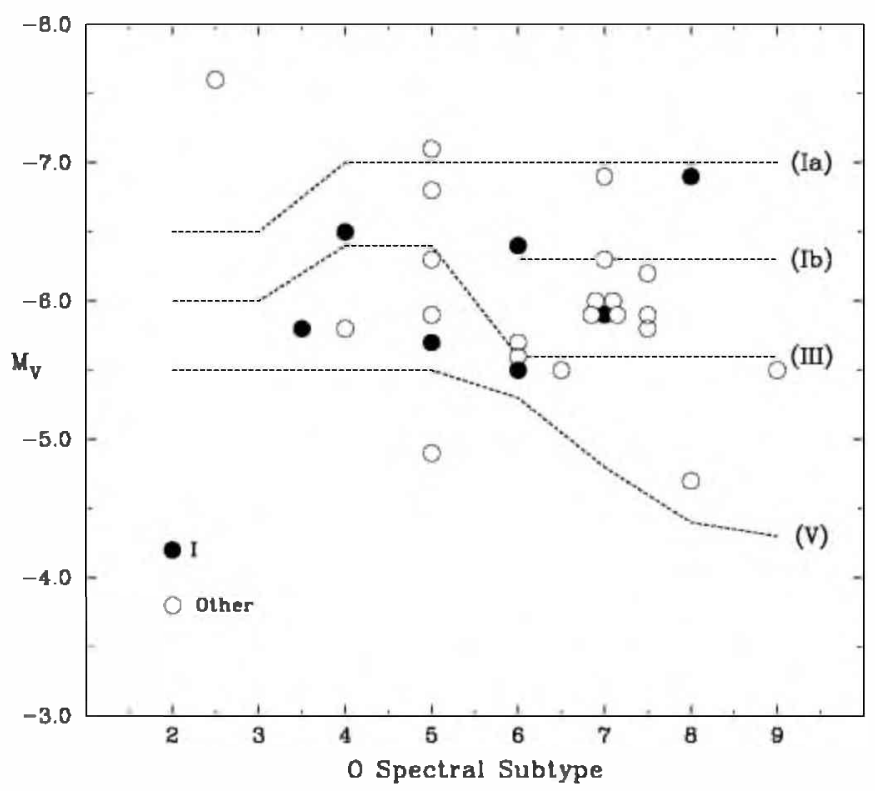

Figure 4. Absolute visual magnitudes of the Onfp sample plotted against spectral types. Filled symbols: luminosity class I; open symbols: no luminosity class assigned. The dashed lines show the calibration for normal spectra by Walborn (1973), but Walborn et al. (2002b) for types O2-O3.

adoption of $R=3$ will yield an absolute magnitude that is too faint if the actual ratio is larger, which it may well be in and around $\mathrm{H}$ II regions as is the case for the most discrepant class I objects N11-20, ST 1-28, and ST 1-93. These issues can be investigated in the future with improved angular resolution or temporal monitoring, and more extensive photometric data and reddening analyses, respectively. However, for the present the most straightforward interpretation of Figure 4 is that the Onfp phenomenon occurs at all luminosities.

\subsection{Rotational Velocities}

Although the resolution of many of the spectrograms is too low to attempt a secure measurement of the rotational broadening $v_{(\mathrm{e})} \sin i$, we can make reasonably accurate determinations of the line-broadening parameter $v \sin i$, in all but six cases for which the resolution and/or $\mathrm{S} / \mathrm{N}$ are inadequate for even the latter. Although processes other than rotation may contribute to the line broadening (e.g., macroturbulence), we expect rotation to be the dominant contributor for the sample of mainly broad-lined stars considered here.

To estimate $v \sin i$ values, the OSTAR2002 model grid (Lanz \& Hubeny 2003) was numerically spun up at $10 \mathrm{~km} \mathrm{~s}^{-1}$ steps, with a standard rotational convolution (e.g., Gray 1992). A gray-atmosphere, linear limb-darkening coefficient of 0.6 was used, but comparison with full surface integrations, accounting for the detailed wavelength dependence of the nonlinear limb darkening across the line profile (similarly to Howarth \& Smith 2001), shows no important differences from these results. Models with LMC abundances were used (Galactic models gave insignificant differences and SMC models none). For each observed spectrum, the rotationally broadened grids were then further convolved with a Gaussian matching the instrumental resolution, and the best-fit model (in $T_{\mathrm{eff}}, \log g$, and $v_{(\mathrm{e})} \sin i$ ) identified as that giving the minimum rms residual over a fixed wavelength range.

Final results for $v \sin i$, included in Table 2, were determined using the 4250-4600 A range, in order to give the temperaturesensitive He lines more leverage with respect to the Balmer 


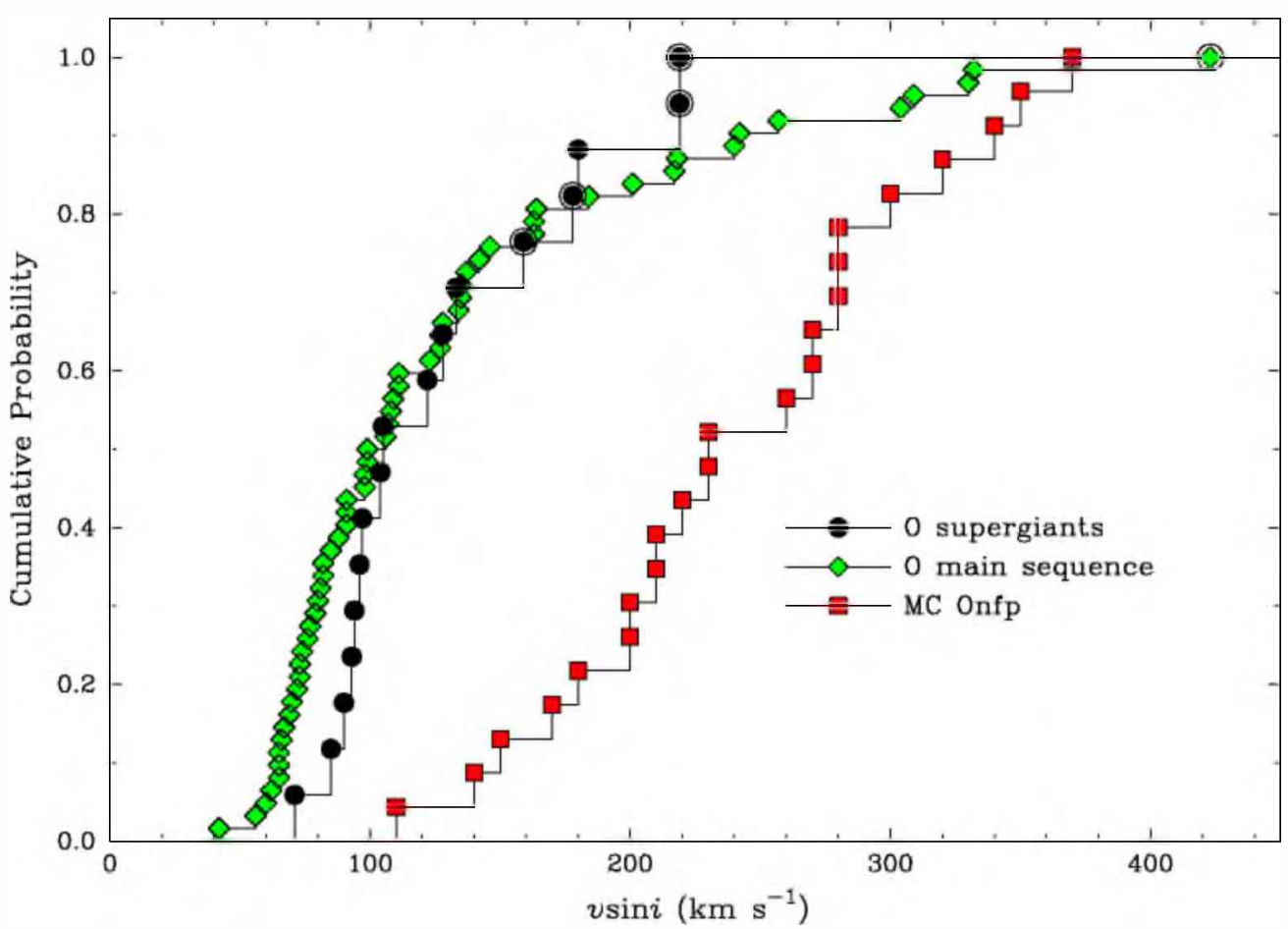

Figure 5. Cumulative probability functions of the line broadening $v \sin i$ for Galactic $\mathrm{O}$ supergiants and main sequence, compared to that of the MC Onfp sample (plus the slow rotator HDE 269702), with symbols as defined in the key; circled Galactic symbols are Onfp spectra.

(A color version of this figure is available in the online journal.)

lines. In practice, the measurements are reasonably insensitive to $T_{\text {eff }}$ and $\log g$, but there is a systematic tendency for the best fits to occur at the lowest model gravities for a given $T_{\text {eff }}$. The generally large $v \sin i$ results are not an artifact of data quality, as some of the largest values are derived from the highresolution UVES data, and lower values are found among the low-resolution spectra.

Comparison of our $v \sin i$ values with those determined by Penny \& Gies (2009) from Far Ultraviolet Spectroscopic Explorer data for six stars in common shows reasonable agreement, with a negligible systematic offset and a median absolute difference of $30 \mathrm{~km} \mathrm{~s}^{-1}$; this number is likely a reasonable upper limit to the uncertainties in our measurements.

Figure 5 compares the cumulative distribution function of $v \sin i$ for the MC Onfp sample (plus the "slow" rotator HDE 269702) with those for Galactic dwarfs and supergiants of spectral types 08 and earlier, that have determinations by Howarth et al. (1997) from International Ultraviolet Explorer data and empirical templates. The Onfp sample differs from both of the latter with $>99.99 \%$ confidence according to a Kolmogorov-Smirnov test, that is, it is comprised of broaderlined spectra. Again, we note that the Onfp class is defined by the He II $\lambda 4686$ profiles, while the line broadening is an independent variable. Penny \& Gies (2009) have found that rotational velocity distributions are very similar between unevolved and evolved stars in the Galaxy, the LMC, and the SMC, as well as among the unevolved stars of the three galaxies. On the other hand, they find evidence that macroturbulent broadening in the evolved stars is lower in the LMC and SMC samples than in the Galaxy. Thus, the large offset between the MC Onfp and the Galactic spectra in Figure 5 is highly significant.

\subsection{Spectral Variations}

Two observations each of N11-20 and Sk $-67^{\circ} 111$ are shown in Figures 6 and 7, respectively. The N11-20
ESO/VLT/FLAMES data are from the work of Evans et al. (2006) and were obtained on two consecutive nights. They display a significant change in the profile of He II $\lambda$ 4686. From further radial-velocity and profile variations in these data, Evans et al. concluded that N11-20 is a binary.

The change in the $\lambda 4686$ profile of $\mathrm{Sk}-67^{\circ} 111$ between two observations 9 months apart is even more dramatic. While the previously unpublished Australian National University (ANU) 2.3 m data have lower resolution and $\mathrm{S} / \mathrm{N}$ in the continuum, there is no doubt that the strong $\lambda 4686$ emission line is single peaked, i.e., this is not an Onfp spectrum. Prior evidence of variability in this spectrum is discussed by Walborn et al. (2002a); the ANU $2.3 \mathrm{~m}$ data parameters are given by Crowther et al. (2002).

The CTIO/Argus observations of A. F. J. Moffat et al. (unpublished) provided extensive temporal coverage specifically to search for spectroscopic binaries. Nightly means from five consecutive nights of ST 1-28 and ST 1-93 are displayed in Figure 8 . Large night-to-night variations in the $\lambda 4686$ profiles are seen, again with non-Onfp single emission peaks on some nights. Analysis of these data by G. Skalkowski (unpublished) has revealed that ST 1-28 is a spectroscopic binary with a period of 2.35 days and a velocity full amplitude of $200 \mathrm{~km} \mathrm{~s}^{-1}$. while ST 1-93 also displays large radial-velocity variations of undetermined period.

These scattered results strongly suggest that systematic monitoring of Onfp spectra is required to understand their nature. In every case with more than one observation, significant variations are found. An initial program on several of these objects was conducted by N. R. Walborn and A. Ahumada during 2008 October and results will be presented in a subsequent publication.

\subsection{Spatial Distribution}

Of course, all of the Onfp spectra correspond to young fields, but most of them are found at the peripheries of clusters and 


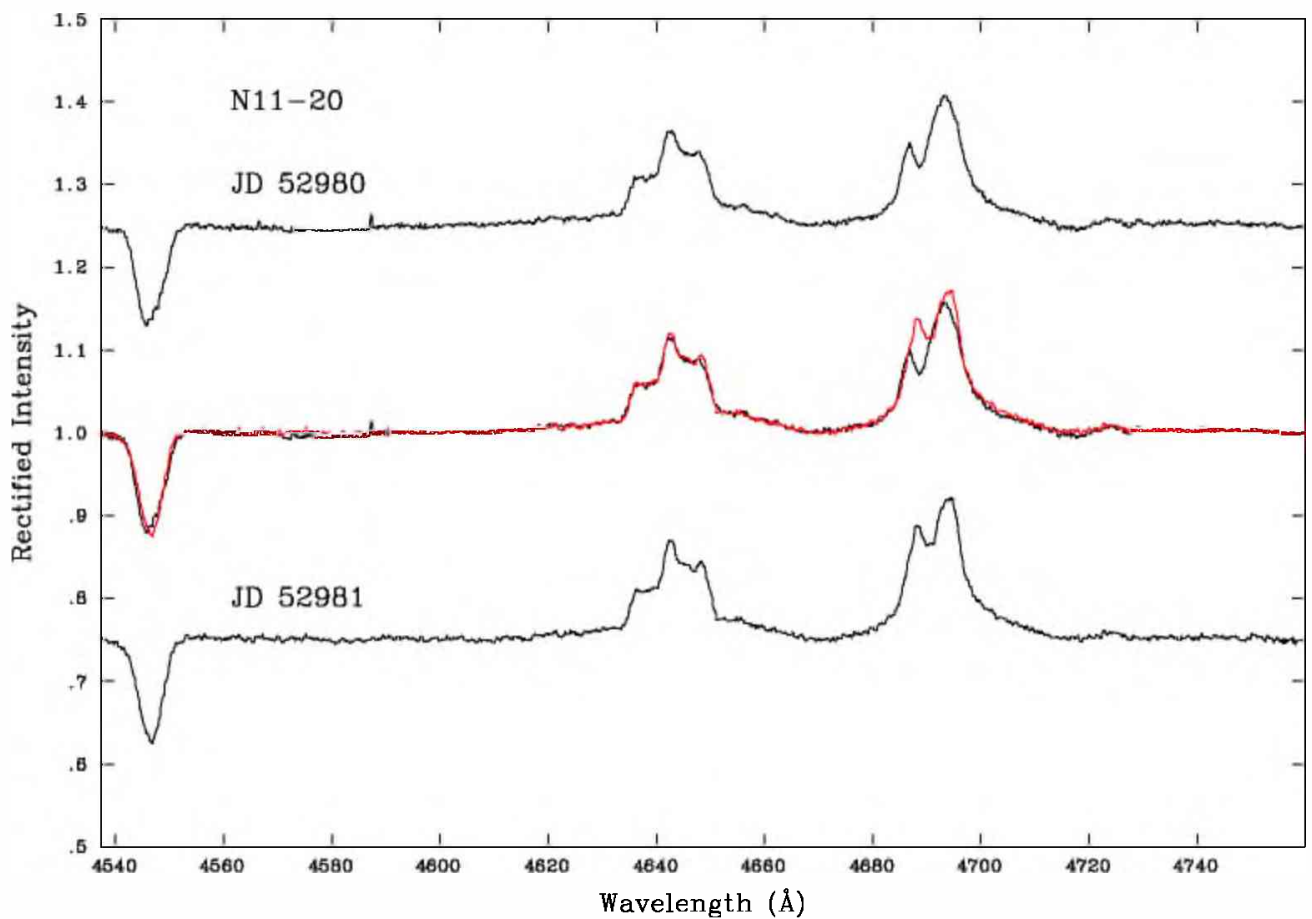

Figure 6. Green region in the spectrum of N11-20 on the two consecutive nights JD 24+ (top and bottom; superimposed in the middle plot). In this and the subsequent figures, observed wavelengths are plotted. The spectral features are He II $\lambda 4541$ absorption, a blend of the broadened N III $\lambda \lambda 4634-4640-4642$ emission lines, and the characteristic Onfp composite profile of He II $\lambda 4686$. The last of these varied significantly between the two nights. Pending detailed fitting, the triple-peaked N III profiles in this and the next figure might indicate that these lines also have Onfp profiles in some spectra.

(A color version of this figure is available in the online journal.)

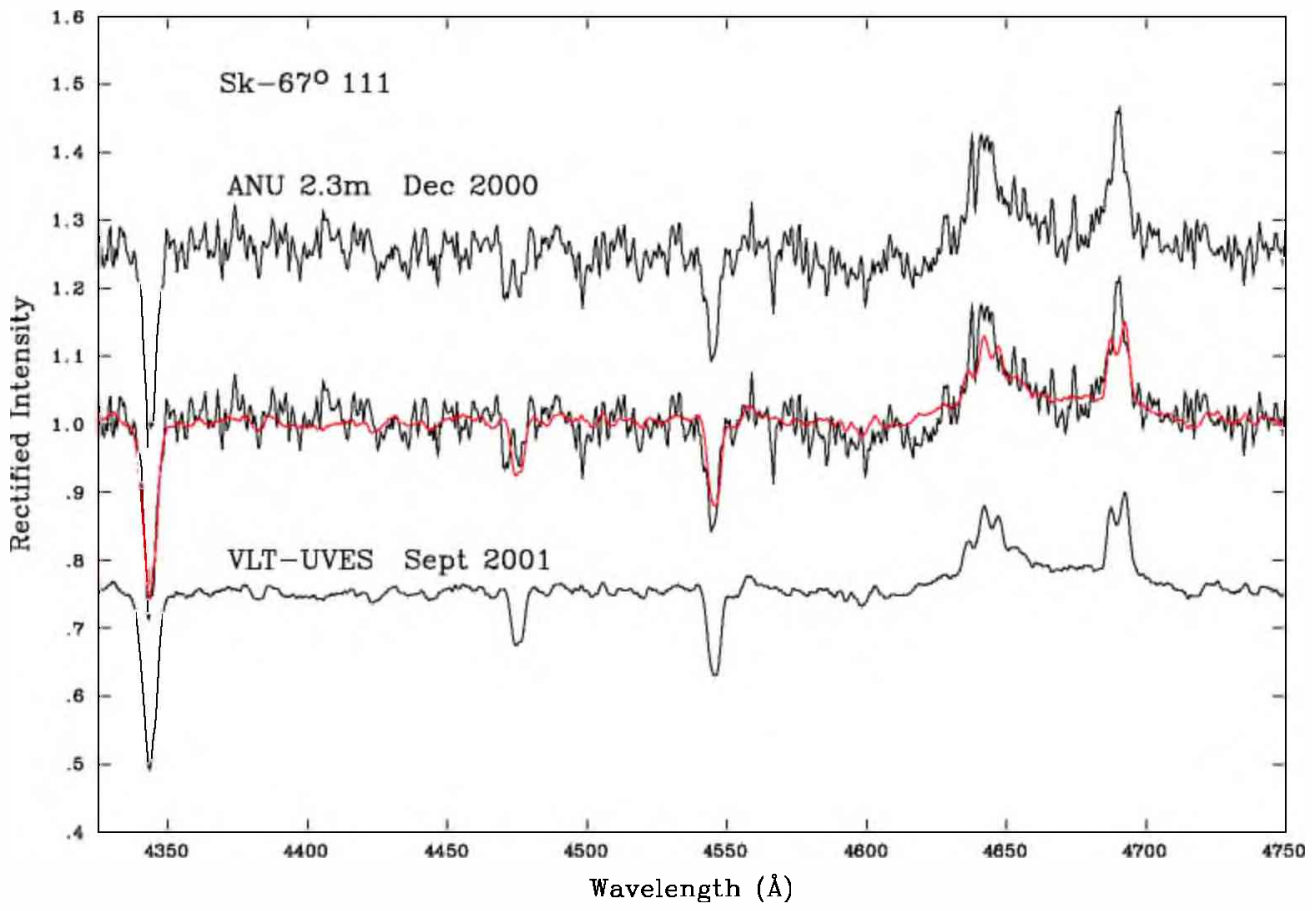

Figure 7. Similar plot to the preceding for $\mathrm{Sk}-67^{\circ} 111$, except that the somewhat wider wavelength range also includes the $\mathrm{H} y \lambda 4340$ and He I $\lambda 4471$ absorption lines, and the two observations are from different instruments used 9 months apart. Despite the data differences, the He II $\lambda 4686$ profile clearly changed in nature; the single-peaked profile at the earlier epoch is not Onfp.

(A color version of this figure is available in the online journal.)

associations, or are even not obviously associated at all. As noted in Section 3.1, only three of them are in bright $\mathrm{H}$ II regions or tight clusters (AA $\Omega$ 30) Dor 333, Brey 73-2A, Parker 841) and two more are in looser subclusterings (ST 1-28, ST 2-53). Interestingly, all five of these are among the earliest spectral types in the sample (Figure 1). That is, the locations of the Onfp class appear analogous to those of normal evolving stars of similar spectral types, and they are not extremely young objects. These results have implications for their origins and nature. 


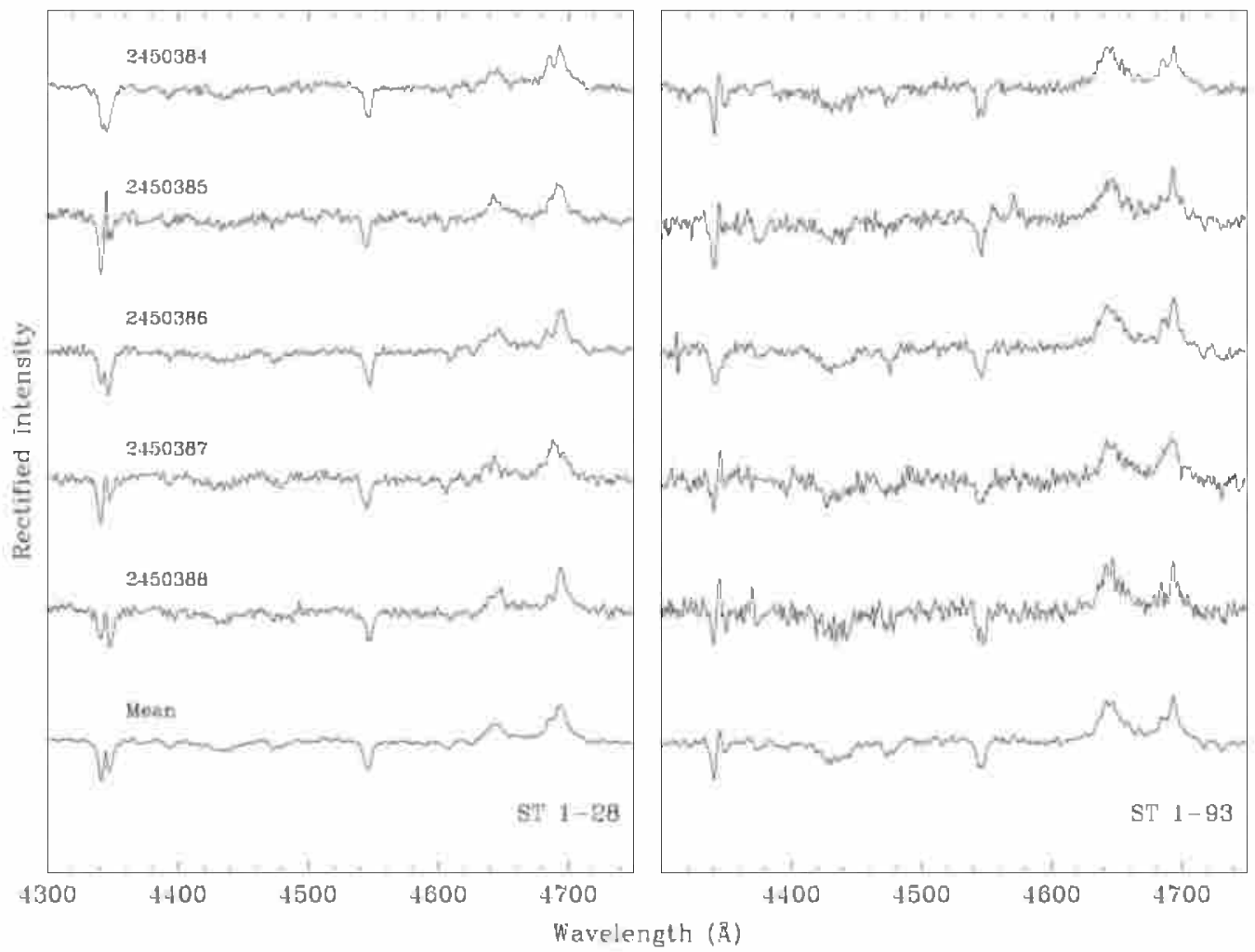

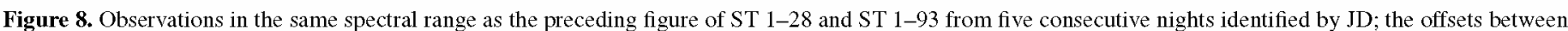

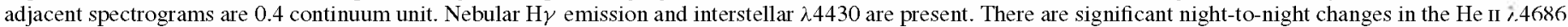

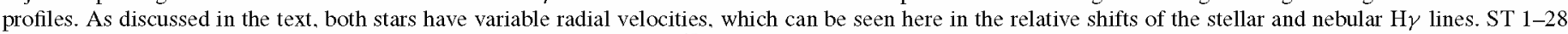
has been found to be a spectroscopic binary with a period of $2.35 \mathrm{~d}$; the $\$ .4686$ profile variations may be correlated.

Alternatively, it could be relevant in this context that both $\zeta$ Pup and $\lambda$ Cep have been determined to be runaways (Mason et al. 1998; Hoogerwerf et al. 2001; and references therein). If that were a common property among the Onfp class, it would be related to their observed spatial distribution in the MCs. Binary mass transfer, spinup of the mass gainer, supernova kicks, and gamma-ray bursts have all been associated with runaways in some models; thus, all of these topics could be interrelated in the Onfp class as further discussed below.

\section{DISCUSSION: POSSIBLE ORIGINS AND DESTINY OF THE Onfp CLASS}

\subsection{Meaning of the Onfp Category}

As a prologue to the discussion, it may be useful to reemphasize that "Onfp" is a description of a (peculiar) spectral morphology, namely reversed He II $\lambda 4686$ emission profiles. In most but not all cases, the latter feature is correlated with line broadening indicative of rapid rotation. In view of this definition, the observed spectral variations that at least in some cases temporarily remove objects from the category, and the a priori possibility that different physical situations may give rise to this general type of $\lambda 4686$ profile, the term "Onfp stars" should be avoided, to prevent any misconception that the spectroscopic category corresponds to a unique, identified physical phenomenon. There may be a predominant mechanism giving rise to the majority of the class, but it remains to be determined whether or not that is true.

\subsection{Binaries}

In a double-lined $O$-type spectroscopic binary, it is in principle possible for (variable) He II $\lambda 4686$ profiles such as those in at least some Onfp spectra to result from two emission features, or an emission line from one star and an absorption line from the other, shifting relative to each other throughout the orbit. In addition, there could be variable emission features arising from mass transfer and/or colliding winds in the system. As reported here, ST 1-28 is a spectroscopic binary, and the Galactic Onfp star HD 152248 is a well-studied, double-lined spectroscopic binary (Sana et al. 2001; Mayer et al. 2008). In particular, Sana et al. showed that in HD 152248, both stellar $\lambda 4686$ features are absorption lines and the emission most likely arises from the colliding winds. However, its Onfp classification has been retained here because it was derived from a single, low-resolution observation (Walborn 1972), as is the case for many of the present $\mathrm{MC}$ sample, so the latter may contain some analogous objects. As also mentioned above, Fariña 35 is an X-ray binary, while ST 1-93 and N11-20 are suspected spectroscopic binaries.

Nevertheless, the general appearance of most Onfp spectra, including several observed at very high resolution, with their comparable broadenings of both absorption and emission lines, is not that of double-lined spectroscopic binaries. Of course, only one observation is available for most of them, and clearly this issue can be resolved only by spectroscopic (and photometric) monitoring. It is possible that multiple mechanisms will apply to some different members of the Onfp class, but it appears likely that a dominant one is relevant to the majority.

\subsection{Rotation}

Characteristically, most of the Onfp class have broadened absorption and emission lines, as shown in the figures and investigated in some detail in Section 3.3. While detailed 
profile fitting at high resolution and $\mathrm{S} / \mathrm{N}$ will be required to establish it, nevertheless rotation is strongly suggested as the line broadening mechanism. The range of $v \sin i$ values found could be intrinsic or caused by inclination effects; the predominance of abnormally large values suggests the latter.

The defining Onfp He II $\lambda 4686$ profiles are reminiscent of the Balmer profiles of Be stars, which naturally suggested the possibility of (hotter) disks in the former. Evidence for line polarization effects in three Galactic Onfp spectra has been found by Harries et al. (2002) and Vink et al. (2009), but the interpretation is complex and any implications for the presence of disks are uncertain; in fact, one of these cases is the binary HD 152248. Moreover, Bouret et al. (2008) have reproduced variable, double-peaked emission profiles in one of these stars, $\zeta$ Pup, with a rotating, clumped wind and no disk.

\subsection{Mergers}

The persistence of rapid rotation in an evolved O-type star with a strong wind presents a problem, since the wind should brake the rotation, as discussed by, e.g., Ekstrom et al. (2008) in addressing the greater relative frequency of rapid rotators and the Be phenomenon among the B stars. Rapid rotation at a late age may be induced by mass transfer (Langer et al. 2008) and/or a merger in a binary system, in which orbital angular momentum is converted to rotation (e.g., Dale \& Davies 2006); such an object may also be a candidate gamma-ray burst progenitor, as they discuss. In this regard, it is interesting that Brey 73-2A lies about 2 mag above the apparent main-sequence turnoff in its compact cluster (Walborn et al. 1999); it could be a blue straggler resulting from a binary merger. Of course, this is a single case, and it could also be simply a result of unresolved multiplicity, although ST 2-53 may be a similar case. The possibility of binary mass transfer and stellar mergers warrants consideration in further analysis of the Onfp phenomenon.

\section{SUMMARY}

We have investigated a sample of $28 \mathrm{Onfp}$ (and related) spectra in the MCs, defined primarily by composite, non-PCygni emission plus absorption profiles in the key He II $\lambda 4686$ line. Their diverse spectral morphology, absolute magnitudes, line broadening, spectral variations, and spatial distribution have been surveyed. The salient results in each area are as follows. There is a range of $\lambda 4686$ profile shapes, but the broadening of emission and absorption lines is uniformly correlated. There is no relationship between the emission-line strengths and the absolute magnitudes in these peculiar objects, as is the case for normal stars. Large, likely rotational line broadening predominates, although a few narrow-lined objects are found; cumulative line-broadening distributions show definitively that the Onfp spectra are drawn from a different parent population than normal spectra. All objects with multiple observations display significant spectral variations, which can even remove some spectra from the Onfp class, i.e., the $\lambda 4686$ emission becomes single peaked, temporarily. Only a few of these objects are located in compact clusters; most lie in the peripheries of associations or even in the (young) field.

Some of the Onfp objects are spectroscopic binaries, including at least one X-ray binary, which may be the cause of the peculiar and variable $\lambda 4686$ profiles in those cases; the current data are inadequate to determine binarity or otherwise for most of them. The most likely hypothesis for the line broadening is rotational, but there is currently no compelling evidence for disks (as in Be stars) as the predominant source of these $\lambda 4686$ profiles; in one Galactic case it has been reproduced by a rotating, clumped wind model. Mass-transfer binaries or stellar mergers are possible origins of these evolved rapid rotators with strong winds, and they merit consideration as gamma-ray burst progenitors.

These results suggest several observational and analytical developments that will contribute to understanding the nature of the Onfp class. Clearly, intensive spectral and photometric monitoring, including radial-velocity measurements, are essential to determine the nature of the endemic variability seen in the limited available coverage. High spectral resolution and $\mathrm{S} / \mathrm{N}$ will be preferable. High spatial-resolution images to search for multiplicity, and more extensive photometric and reddening analyses, will improve the accuracy of the absolute magnitudes (although a gap between spatially and kinematically determined multiplicities in the MCs will remain for the immediate future). Quantitative spectral analyses of high-quality data will establish (or otherwise) the rotational interpretation of the line broadening and derive chemical abundances (especially of $\mathrm{CNO}$ ) and other parameters essential for a definitive physical model (or models) of the Onfp spectral category.

We thank the AAO, ESO, CTIO, and LCO staffs for their support in the acquisition of the data reported here. Publication support was provided by the STScI Director's Discretionary Research Fund. A.F.J.M. and N.S.L. thank the National Sciences and Engineering Research Council of Canada and the Fonds Quebecois de la Recherche sur la Nature et les Technologies for financial assistance. R.H.B. acknowledges partial support from Universidad de La Serena Project DIULS CD08102. We thank the anonymous referee for some useful comments.

\section{REFERENCES}

Ardeberg, A. 1980, A\&AS, 42, 1

Azzopardi, M., \& Vigneau, J. 1982, A\&AS, 50, 291

Bouret, J.-C., Hillier, D. J., \& Lanz, T. 2008, in IAL Symp. 250, Massive Stars as Cosmic Engines, ed. F. Bresolin, P. A. Crowther, \& J. Puls (Cambridge: Cambridge Univ. Press). 526

Breysacher, J., Azzopardi, M., \& Testor, G. 1999, A\&AS, 137, 117

Conti, P. S., \& Frost, S. A. 1974, ApJ, 190, L137

Conti, P. S.. \& Leep, E. M. 1974, ApJ, 193, 113

Conti, P. S., \& Niemela, V. S. 1976, ApJ, 209, L37

Crowther, P. A., Hillier, D. J., Evans, C. J., Fullerton, A. W., De Marco, O., \& Willis. A. J. 2002. ApJ. 579, 774

Dale, J. E., \& Davies, M. B. 2006, MNRAS, 366, 1424

De Becker, M., \& Rauw, G. 2004, A\&A. 427, 995

Ekstrom. S., Meynet. G., Maeder, A.. \& Barblan. F. 2008, A\&A. 478, 467

Evans, C. J., Howarth, I. D., Irwin, M. J., Burnley, A. W., \& Harries, T. J. 2004, MNRAS, 353, 601

Evans, C. J., Lennon. D. J., Smartt, S. J., \& Trundle, C. 2006, A\&A, 456, 623

Fariña, C., Bosch, G. L., Morrell, N. I., Barbá, R. H., \& Walborn, N. R. 2009, AJ, 138,510

Gray, D. F. 1992. The Observation and Analysis of Stellar Atmospheres (Cambridge: Cambridge Univ. Press)

Harries, T. J., Howarth, I. D., \& Evans, C. J. 2002, MNRAS, 337, 341

Heap, S. R., Lanz, T., \& Hubeny, I. 2006, ApJ, 638, 409

Henize, K. G. 1956, ApJS, 2, 315

Henrichs, H. 1991, in Rapid Variability of OB Stars: Nature and Diagnostic Value, ed. D. Baade (Garching: ESO). 199

Hoogerwerf, R., de Bruijne, J. H. J., \& de Zeeuw, P. T. 2001, A\&A, 365, 49

Howarth, I. D., Siebert, K. W., Hussain. G. A. J., \& Prinja, R. K. 1997, MNRAS, 284,265

Howarth, I. D.. \& Smith, K. C. 2001, MNRAS, 327, 353

Isserstedt, J. 1975, A\&AS, 19, 259

Isserstedt, J. 1979, A\&AS, 38, 239

Kendall, T. R., Dufton, P. L., \& Lennon, D. J. 1995, A\&A, 298, 489

Kendall. T. R., Dufton. P. L., \& Lennon. D. J. 1996. A\&A. 310. 564 
Langer, N., Cantiello, M.. Yoon, S.-C., Hunter. I., Brott, I., Lennon. D., de Mink. S.. \& Verheijdt. M. 2008. in IAU Symp. 250. Massive Stars as Cosmic Engines, ed. F. Bresolin, P. A. Crowther, \& J. Puls (Cambridge: Cambridge Univ. Press), 167

Lanz. T., \& Hubeny, I. 2003, ApJS, 146, 417

Lennon. D. J. 1997. A\&A. 317. 871

Maíz Apellániz, J., Walborn. N. R.. Galué, H. A.. \& Wei, L. H. 2004, ApJS 151,103

Mason, B. D., Gies, D. R., Hartkopf, W. I., Bagnuolo, W. G., Jr., ten Brummelaar, T., \& McAlister, H. A. 1998, ApJ, 115, 821

Massey, P. 2002. ApJS. 141. 81

Massey, P., Waterhouse, E., \& DeGioia-Eastwood, K. 2000, AJ, 119. 2214

Mayer, P., Harmanec, P., Nesslinger, S., Lorenz, R., Drechsel, H., Morrell, N. \& Wolf, M. 2008, A\&A, 481, 183

Mihalas, D. M., \& Conti. P. S. 1980, ApJ, 235, 515

Moffat, A. F. J., \& Michaud, G. 1981, ApJ, 251, 133

Parker, J. Wm. 1993, AJ, 106, 560

Penny, L. R., \& Gies, D. R. 2009, ApJ, 700, 844

Rauw, G., De Becker. M., \& Vreux. J.-M. 2003. A\&A. 399. 287

Sana, H., Rauw. G.. \& Gosset. E. 2001, A\&A. 370. 121

Sanduleak, N. 1969, AJ, 74, 877

Sanduleak, N. 1970, CTIO Contr 89

Schild. H., \& Testor. G. 1992, A\&AS, 92, 729

Testor. G.. \& Niemela. V. 1998, A\&AS, 130, 527
Udalski, A., Szymanski, M.. Kubiak. M., Pietrzyński, G.. Soszyński. I. Woźniak. P.. \& Zebruñ. K. 2000. Acta Astron.. 50. 307

van der Meer. A., Kaper. L., van Kerkwijk, M. H.. Heemskerk, M. H. M., \& van den Heuvel, E. P. J. 2007, A\&A, 473, 523

Vink, J. S., Davies, B., Harries, T. J., Oudmaijer, R. D., \& Walborn. N. R. 2009. A\&A. 505. 743

Walborn, N. R. 1971, ApJS, 23, 257

Walborn, N. R. 1972, AJ, 77, 312

Walborn, N. R. 1973, AJ, 78, 1067

Walborn, N. R. 1982, AJ, 87, 1300

Walborn. N. R. 2001, in ASP Conf. Ser., 242. Eta Carinae \& Other Mysterious Stars, ed. T. Gull. S. Johansson, \& K. Davidson (San Francisco, CA: ASP). 217

Walborn, N. R. 2009, in Stellar Spectral Classification, ed. R. O. Gray \& C. Corbally (Princeton, NJ: Princeton Univ. Press), 66

Walborn, N. R., \& Blades, J. C. 1997, ApJS, 112, 457

Walborn, N. R., Drissen, L., Parker, J. Wm., Saha, A., MacKenty, J. W., \& White, R. L. 1999, AJ, 118, 1684

Walborn, N. R.. \& Fitzpatrick. E. L. 1990, PASP. 102, 379

Walborn. N. R.. Fullerton. A. W. Crowther. P. A.. Bianchi. L.. Hutchings, J. B.. Pellerin, A., Sonneborn, G., \& Willis, A. J. 2002a, ApJS, 141, 443

Walborn, N. R., Lennon. D. J., Heap, S. R., Lindler, D. J., Smith. L. J., Evans, C. J., \& Parker, J. Wm. 2000, PASP, 112, 1243

Walborn, N. R., et al. 2002b, AJ, 123, 2754

Zaritsky, D.. Harris. J., Thompson. I. B.. \& Grebel. E. K. 2004. AJ. 128, 1606 